\title{
THE DISORDERING EFFECT ON THE ONE-DIMENSIONAL APPERIODIC OPTICAL SUPER-LATTICE IN MULTI-FREQUENCY SHG
}

\author{
MAJID NEMATI \\ Yerevan State University, Alex Manoogian 1, 0025, Yerevan, Armenia \\ m.nemati55@gmail.com
}

\begin{abstract}
A multi-frequency second harmonic generation (SHG) in one dimensional nonlinear aperiodic optical superlattice in the presence of linear spacers is considered. The effect of random fluctuations in the length of each layer on the output efficiency of SHG process is investigated, and the favorable percentage of fluctuation in the fabrication of aperiodic optical superlattice is obtained. Comparing perfect and fluctuated aperiodic structures, the researcher realized the decrease in the amount of output efficiency with the increase of fluctuation in the linear-nonlinear aperiodic crystals.
\end{abstract}

Keywords: Nonlinear Optics; Harmonic generation and multiple wavelengths; Quasi-phasematching.

\section{Introduction}

Phase matching (PM) between interacting waves has a critical role on the output efficiency of the nonlinear optical parametric processes like second harmonic generation (SHG) ${ }^{1}$. The PM condition can be maintained by using birefringence crystal or utilizing Multi layered structure which is known as quasi-phase-matching (QPM) technique. Layered structures have a property in which the sign of second order nonlinear susceptibility reverses periodically in the length of crystal ${ }^{2}$. Recently, the combination of linear and nonlinear domains in the layered structure has been studied in SHG and down conversion (DC) processes. The combination of linear-nonlinear domains improves PM condition at the nonlinear interactions. In other words, linear layers have the role of compensator in the designed structure ${ }^{3-5}$. Different gratings are used to prepare new frequencies in nonlinear interactions through which one or multi new frequencies are obtained simultaneously ${ }^{1,6}$. It is possible to maintain PM condition for the creation of multi- frequency (MF) in SHG by using different methods. MF is a process in which the $\mathrm{PM}$ is satisfied for more than one wave vectors with the appropriate definition of optical super-lattice. MF process is utilized in the creation of new laser sources for medical sensing applications and agricultural analyses ${ }^{7}$. Recently, structures such as Fibonacci super-lattice, quasi periodic super-lattice and aperiodic optical super-lattice (AOS) are used to satisfy QPM condition for achieving MF process in one-dimension ${ }^{7-9}$. A random 
arrangement of domains is considered along the crystal in the AOS structure such that the domain size is an integer multiple of a unit block ${ }^{6}$. The AOS structures are studied in one and two-dimension in more details ${ }^{6,9}$ and 10 . Construction of these kinds of structures can be performed by using different simulation and optimization algorithms like Simulated Annealing (SA) in one and two dimensional structures ${ }^{6,10}$. The presence of fluctuation in the parameters which affect the output efficiency is unavoidable. One of these parameters is the effect of fluctuations on the length of periodic and aperiodic linear-nonlinear configurations in the AOS structure, ${ }^{4,9}$. Recently, the problem of fluctuation in the arrangement of layers and the position of boundaries in SHG and DC is studied which shows some unusual properties $4,5,11$ and 12 . Therefore, the effect of fluctuation on the length of layers has noticeable effect on the efficiency of frequency mixing nonlinear process.

In this Paper, the AOS structure in the presence of linear media as a spacer in the nonlinear lattice in SHG process with disordered domains is theoretically studied and the role of fluctuation in the AOS structure on the output efficiency in the PZNZ model (periodic combination of positive, zero and negative susceptibilities [6]) is investigated. The organization of the paper is as follows:

In section two, the perfect structure for SHG process in the PZNZ model has been illustrated. Then, the SA method is used to attain an optimal AOS in the one-dimensional PZNZ structure. Next, in section three, the effect of random fluctuation of position of boundaries of segments in the PZNZ structure on the reduced effective nonlinear coefficient is studied. Finally, in section four the conclusion is drawn.

\section{Theoretical Approach}

The combination of linear and nonlinear domains with the nonlinear segments on the basis of $\mathrm{LiNbO}_{3}$ in which the linear segments are considered as spacers in the PZNZ structure is considered. The AOS structure is appropriate for maintaining multi-QPM which is used as a new source of MF conversion in SHG process. In order to have almost high amount of efficiency in MF conversion process an arrangement of linear and nonlinear layers is required which can be utilized by the optimization methods like $\mathrm{SA}^{6,9}$ and 10 . PM condition improves by adding linear spacers in order to increase the efficiency of MF SHG process. For this purpose, the results of Ref. 4 are considered to illustrate theoretical method in SHG in the presence of linear segments in the periodic nonlinear structure which is called PZNZ model. In this model, nonlinear layers are alternated by linear layers with the zero second order nonlinear susceptibility, and the sign of susceptibility for nonlinear segments is aperiodically changed through the media (Fig. 1). 


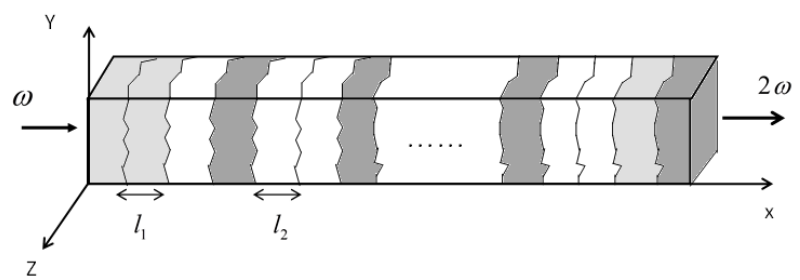

Fig. 1. The aperiodic PZNZ structure in which light gray and dark gray strips represent positive and negative susceptibilities in the nonlinear media with the length of $l_{1}$ and white strips belongs to linear segments with the length of $l_{2}$.

A laser beam with frequency $\omega$ which is propagated through the x-axes in the AOS structure is considered. By assuming slowing-wave variation of the field amplitude, we can represent the output efficiency as follow:

$$
\eta=\frac{I_{2 \omega}}{I_{\omega}}=\frac{\omega^{2}\left|E_{\omega}\right|^{2} L^{2}}{4 n_{\omega} n_{2 \omega} c^{2}} d^{2} .
$$

where $d$ is the second order nonlinear coefficient of the sample, $I_{\omega}$ and $I_{2 \omega}$ are the input and output intensities of the process respectively, $\omega$ is the input wave frequency, $n_{1}$ and $n_{2}$ are refractive indexes for fundamental and SH waves respectively, $\mathrm{c}$ is the speed of light, $L$ is the total length of crystal and $E_{\omega}$ is the amplitude of input wave. The second order nonlinear coefficient is denoted as:

$$
d=\left|\frac{1}{L} \int_{0}^{L} \chi^{(2)}(x) e^{-i \Delta k x} d x\right|
$$

By considering the poling function $\mathrm{g}(\mathrm{x})$ that takes values of $(1$ or -1$)$ and $(0)$ for nonlinear and linear domains respectively, we can express the modified effective nonlinear coefficient as follows ${ }^{4}$ :

$$
d_{\text {eff }}=\left|\frac{1}{L \Delta k^{\prime}} \sum_{n=1}^{N} g_{n+1} e^{\left(-i \Omega_{n}\right)}\left[e^{\left(-i \Delta k^{\prime} x_{n}\right)}-e^{\left(-i \Delta k^{\prime} x_{n-1}\right)}\right]\right| .
$$

where $\Omega_{\mathrm{n}}=\sum_{\mathrm{r}=0}^{\mathrm{n}-1}(-1)^{\mathrm{r}+1} \mathrm{x}_{\mathrm{r}} \gamma, x_{r}$ are the positions of domain boundaries and $\gamma=\Delta k^{\prime}-\Delta k^{\prime \prime}$. The wave vector mismatch for the nonlinear and linear segments are $\Delta k^{\prime}$ and $\Delta k^{\prime \prime}$, respectively. The mismatch functions are the same in all domains. The length of the crystal is $\mathrm{L}=(\mathrm{N} / 2) *\left(l_{1}+l_{2}\right)$, the number of domains is $N$, and $l_{1}, l_{2}$ are the length of nonlinear and linear domains respectively ${ }^{6}$.

Solving the phase mismatch problem in nonlinear periodical crystals is done by reversing the domain orientation such that the constructive interference happens because of the occurred phase adjusting. In aperiodic structure, phase-matching depends on solving an inverse source problem ${ }^{9}$ which can be solved with the help of mathematical optimization processes over the output efficiency to have the desired rations of output efficiency. One of the most appropriate optimization methods in this case is SA algorithm 
which is a probabilistic method for finding the global minimum of an objective function that may possess several local minima ${ }^{13}$. The consideration of an objective function " $F$ " is an important factor in this optimization method in which F should be minimized and the appropriate arrangement of the domain orientation in the crystal can be determined. For example, five wavelengths of $0.972,1.082,1.283,1.364$, and $1.5687 \mu \mathrm{m}$ are chosen. The lengths of nonlinear and linear segments are $l_{1}=3 \mu \mathrm{m}, l_{2}=0.1 \mu \mathrm{m}$ and the number of layers $N=2000$ are chosen to have MF SHG process for the assumed wavelengths. The dispersion of the refractive index of the material is evaluated through Sellmeier formula ${ }^{14}$ and the objective function in the SA optimization technique is chosen as:

$$
F=\sum_{i}\left|d^{(0)}-d_{e f f}\left(\lambda_{i}\right)\right|+A\left\{\max \left(d_{e f f}\left(\lambda_{i}\right)\right)-\min \left(d_{e f f}\left(\lambda_{i}\right)\right)\right\} .
$$

where the symbol max, min represents maximum and minimum value among all the quantities included in the parenthesis. $A$ is the constant amount to balance each $d_{\text {eff }}$ and $d^{(0)}$ is the constant amount obtained from experiment. The dispersion relation of the refractive index at the fundamental and SHG wavelengths are calculated at the room temperature. The number of sampling wavelengths is 800 between the range of 0.8 and $1.6 \mu \mathrm{m}$.

\section{Results and Discussion}

By considering the aperiodic configuration in the SHG process in the PZNZ model, I managed to review the behavior of the $d_{\text {eff }}$ with respect to various conditions of the structure in more details. For this purpose, the properties of the $d_{e f f}$ in the perfect and fluctuated PZNZ configuration are considered. The gray-scale diagram of the AOS is represented in Fig. 2 where the black, white and gray strips show the negative, positive and zero layers respectively ${ }^{6}$.

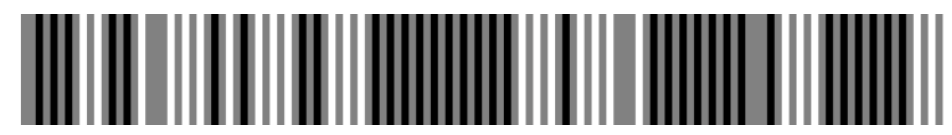

Fig. 2. The gray-scale diagram of PZNZ. The black, white and gray strip shows the negative, positive and zero layers respectively.

To concentrate on the role of fluctuation in the AOS structure which is obtained by using SA optimization algorithm, a fluctuation in the length of segments of the AOS structure is considered which is illustrated in Fig. 3. For this purpose, the random fluctuation in the lengths of each segment is considered and its effect on the $d_{\text {eff }}$ is achieved. To consider fluctuation in length of each segment a Gaussian random number generator is used (between 0 and 1 with equal probability) ${ }^{11}$. The fluctuation functions in Figs. $3(a, b, c)$ are $0.05 \times \cos (2 \pi \times R), 0.1 \times \cos (2 \pi \times R)$ and $0.2 \times \cos (2 \pi \times R)$ respectively ( $\mathrm{R}$ is a Gaussian random number) where the length of nonlinear and linear layers are $l_{1}=3 \mu m$ and $l_{2}=0.1 \mu m$ respectively. 

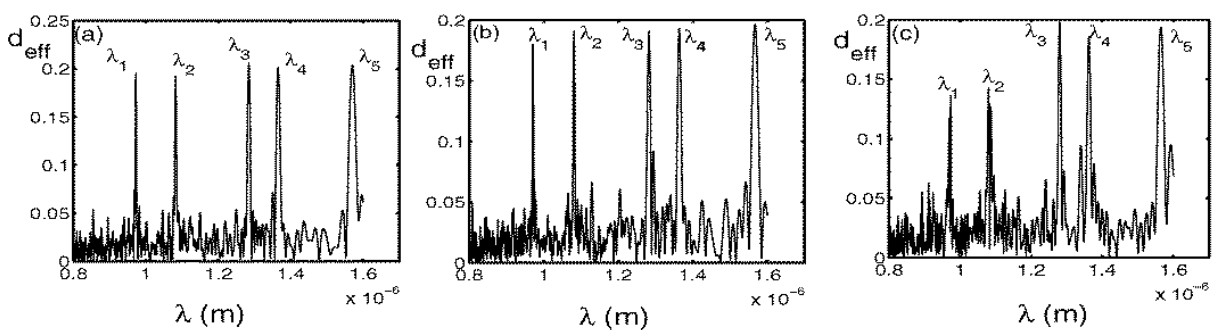

Fig. 3.The behavior of the reduced effective nonlinear coefficient in constructed fluctuated AOS structure in PZNZ model. (a) The assumed amount of fluctuation is $5 \%$ of each designed domain length; (b) The assumed amount of fluctuation is $10 \%$ of each designed domain length; (c) The assumed amount of fluctuation is $20 \%$ of each designed domain length. The assumed lengths of nonlinear and linear segments are $l_{1}=3 \mu \mathrm{m}$ and $l_{2}=0.1 \mu m$ respectively.

Here the influence of fluctuation in the length of segments of PZNZ on SHG process is reviewed. The maximum values of fluctuation in Figs. 3(a, b, c) are 5, 10 and 20 percent of the designed length of segments respectively. It is evident from the Fig. 3 that various fluctuations in the length of segments in the same structure demonstrate remarkable results. As the amount of fluctuation rises, the maximum value of $d_{\text {eff }}$ for five fundamental wavelengths decreases, such that the mean value of $d_{e f f}$ reduces. With regard to the data in Fig. 3(c), it is realized that increasing the percentage of fluctuation causes the maximum values of $d_{\text {eff }}$ to be obtained in higher wavelengths. It is deduced that the reducing rate in the value of $d_{\text {eff }}$ in the perfect PZNZ model in Ref. 6 is slower than that of the fluctuated one. Another result is that the effect of fluctuation in the PZNZ model is less than that of the periodic poled nonlinear crystal which means that the presence of linear media in the AOS structure helps to satisfy phase matching condition and the effect of linear media comes out as a lower decrease rate of the $d_{\text {eff }}$ in the PZNZ structure. It is concluded that the presence of linear segments can be useful in AOS structure to protect the system from fluctuation in the lengths of layers during the fabrication of these crystals.

\section{Conclusion}

In conclusion, by considering arrangements of nonlinear and linear layers and using the SA method, the optimized aperiodic optical superlattice is designed to consider the influence of fluctuation in the length of domains in multiple frequency amplification on the amount of the reduced effective nonlinear coefficient $\left(d_{e f f}\right)$. By concentrating on the effect of fluctuation on the length of domains in the PZNZ structure, I conclude that the value of $d_{\text {eff }}$ decreases as the amount of fluctuation increases. Also, it is realized that as the percentage of fluctuation increases, the $d_{e f f}$ in the PZNZ model has lower reduction rate, in comparison with periodic poled nonlinear crystal ${ }^{9}$ because of the presence of linear segments in the PZNZ model. 


\section{References}

1. M. M. Fejer, G. A. Magel, D. H. Jundt and R. L. Byer, IEEE J. Quantum Electron. 28, 2631 (1992).

2. D. S. Hum, M. M. Fejer, C. R. Phys. 8(2), 180 (2007).

3. G. Kryuchkyan and D. Antonosyan, Modern optics and photonics: Atoms and structured media, World Scientific, Singapore, 131 (2010).

4. M. Rezaei, R. Kheradmand, G. Yu. Kryuchkyan and M. Nemati, J. Nonlinear Optical Physics \& Materials. 20, 23 (2011).

5. R. Kheradmand, M. Rezaei, G. Yu. Kryuchkyan, M. Nemati and A. Razmi, Phys. Status. Solidi A. 208, 1849 (2011).

6. M. Nemati, R. Kheradmand, M. Rezaei, G. Yu. Kryuchkyan, N. Sang Nour Pour and M. Goalipour, Multipe quasi-phase-matching in one dimensional aperiodicaly poled optical superlattice, to appear in J. Phys. B: At. Mol. Opt. Phys.

7. S. N. Son, J. J. Song, J. U. Kang and C. S. Kim, Sensors 11, 6125 (2011).

8. L. Chen, X. Chen, Y. Chen and Y. Xia, Phys. Lett. A. 349, 484 (2006).

9. B. Y. Gu, Y. Zhang and B. Z. Dong, J. Appl. Phys. 87, 7629 (2000).

10. N. Sang Nour Pour, M. Nemati, M. Goalipour, R. Kheradmand, M. Rezaei and G. Kryuchkyan, Multi-Frequency SHG process in the presence of disordered two-dimensional aperiodic optical superlattice, in Proc. Int. Conf. Laser Physics 2011(Ashtarak, Armenia 2011), p. 80.

11. X. Vidal and J. Martorell, Phys. Rev. Lett. 97, 013902 (2006).

12. D. Antonosyan, M. Nemati and G. Kryuchkyan, Proc. SPIE 8414, 84140T (2011).

13. S. Kirkpatrick, C. D. Gelat and M. P. Vecchi, Science 220, 671 (1983).

14. J. P. Meyn and M. M. Fejer, Opt. Lett. 22, 1214 (1997). 